\title{
Spine stereotactic radiosurgery with concurrent tyrosine kinase inhibitors for metastatic renal cell carcinoma
}

\author{
Jacob A. Miller, BS, ${ }^{1}$ Ehsan H. Balagamwala, MD, ${ }^{2}$ Lilyana Angelov, MD, ${ }^{3,4}$ John H. Suh, MD, ${ }^{2,4}$ \\ Brian Rini, MD, ${ }^{5}$ Jorge A. Garcia, MD, ${ }^{5}$ Manmeet Ahluwalia, MD, ${ }^{4,5}$ and Samuel T. Chao, MD ${ }^{2,4}$ \\ ${ }^{1}$ Cleveland Clinic Lerner College of Medicine, Departments of ${ }^{2}$ Radiation Oncology, ${ }^{3}$ Neurosurgery, and ${ }^{5}$ Medical Oncology, \\ Taussig Cancer Institute; and ${ }^{4}$ Rose Ella Burkhardt Brain Tumor and Neuro-Oncology Center, Cleveland Clinic, Cleveland, Ohio
}

OBJECT Systemic control of metastatic renal cell carcinoma (mRCC) has substantially improved with the development of VEGF, mTOR, and checkpoint inhibitors. The current first-line standard of care is a VEGF tyrosine kinase inhibitor (TKI). In preclinical models, TKIs potentiate the response to radiotherapy. Such improved efficacy may prolong the time to salvage therapies, including whole-brain radiotherapy or second-line systemic therapy.

As the prevalence of $\mathrm{mRCC}$ has increased, the utilization of spine stereotactic radiosurgery (SRS) has also increased. However, clinical outcomes following concurrent treatment with SRS and TKIs remain largely undefined. The purpose of this investigation was to determine the safety and efficacy of TKIs when delivered concurrently with SRS. The authors hypothesized that first-line TKIs delivered concurrently with SRS significantly increase local control compared with SRS alone or TKIs alone, without increased toxicity.

METHODS A retrospective cohort study of patients undergoing spine SRS for mRCC was conducted. Patients undergoing SRS were divided into 4 cohorts: those receiving concurrent first-line TKI therapy (A), systemic therapy-naïve patients (B), and patients who were undergoing SRS with (C) or without (D) concurrent TKI treatment after failure of firstline therapy. A negative control cohort $(E)$ was also included, consisting of patients with spinal metastases managed with TKIs alone. The primary outcome was 12-month local failure, defined as any in-field radiographic progression. Multivariate competing risks regression was used to determine the independent effect of concurrent first-line TKI therapy upon local failure.

RESULTS One hundred patients who underwent 151 spine SRS treatments (232 vertebral levels) were included. At the time of SRS, $46 \%$ were receiving concurrent TKI therapy. In each SRS cohort, the median prescription dose was 16 Gy in 1 fraction. Patients in Cohort A had the highest burden of epidural disease $(96 \%, p<0.01)$.

At 12 months, the cumulative incidence of local failure was $4 \%$ in Cohort A, compared with $19 \%-27 \%$ in Cohorts B-D and $57 \%$ in Cohort $E(p<0.01)$. Multivariate competing risks regression demonstrated that concurrent first-line TKI treatment (Cohort A) was independently associated with a local control benefit (HR 0.21, $p=0.04$ ). In contrast, patients treated with TKIs alone (Cohort E) experienced an increased rate of local failure (HR 2.43, $p=0.03$ ). No toxicities of Grade 3 or greater occurred following SRS with concurrent TKI treatment, and the incidence of post-SRS vertebral fracture (overall $21 \%$ ) and pain flare (overall 17\%) were similar across cohorts.

CONCLUSIONS The prognosis for patients with $\mathrm{mRCC}$ has significantly improved with TKIs. The present investigation suggests a local control benefit with the addition of concurrent first-line TKI therapy to spine SRS. These results have implications in the oligometastatic setting and support a body of preclinical radiobiological research.

http://thejns.org/doi/abs/10.3171/2016.4.SPINE16229

KEY WORDS spine radiosurgery; tyrosine kinase inhibitors; renal cell carcinoma; oncology

$\mathrm{S}$ YSTEMIC control of metastatic renal cell carcinoma (mRCC) has substantially improved following the development of antiangiogenic tyrosine kinase inhibitors (TKIs). ${ }^{15-17}$ A progression-free survival benefit of first-line sunitinib over interferon-alfa was reported in a
Phase III randomized trial, with recent data demonstrating similar efficacy for pazopanib. ${ }^{15-17}$ Moreover, these agents demonstrate superior objective response rates compared with conventional cytokine therapies and may be administered orally. ${ }^{16,17}$

ABBREVIATIONS mRCC = metastatic renal cell carcinoma; SBRT = stereotactic body radiotherapy; SRS = stereotactic radiosurgery; TKI = tyrosine kinase inhibitor . SUBMITTED February 25, 2016. ACCEPTED April 27, 2016.

INCLUDE WHEN CITING Published online July 8, 2016; DOI: 10.3171/2016.4.SPINE16229. 
Among patients with RCC, the most common sites of metastasis are the lungs, bone, liver, and brain. ${ }^{12,14}$ RCC is a classically radioresistant tumor, and higher doses of radiation are generally required for palliation and local control., ${ }^{3,19,26,27}$ For patients with metastases to the spine, stereotactic radiosurgery (SRS) offers palliation, prophylaxis against neurological compromise, and may be used to delay the initiation of systemic therapy. Several institutions have recently reported outcomes following radiotherapy with concurrent or adjuvant targeted therapies, hypothesizing that these agents may improve local control of treated sites. ${ }^{1,10,24,25,28}$ Such improved efficacy may prolong the time to salvage therapies, including whole-brain radiation therapy or second-line systemic therapy. ${ }^{1,25}$ In murine and in vitro models, TKIs have been shown to potentiate the response to radiation. ${ }^{13,23,30}$ With fewer adverse effects than cytokine therapies, TKIs are attractive agents for concurrent administration with SRS.

Although uncontrolled outcomes following SRS with concurrent TKI treatment have been reported, no comparative outcomes exist, and the safety and efficacy of concurrent therapy remains unclear. ${ }^{1,10,24,25,28}$ It is unknown whether these favorable outcomes are attributable to TKIs alone, SRS alone, or concurrent therapy. Therefore, we sought to compare local control of spinal metastases treated with SRS and TKIs, stratifying by failure of prior systemic therapy. We hypothesized that patients undergoing SRS with concurrent first-line TKI therapy achieve superior local control compared with therapy-naïve patients, those in whom first-line systemic therapy has failed, and those treated with TKIs alone.

\section{Methods \\ Patient Selection}

An institutional review board-approved database was retrospectively queried for all patients who underwent spine SRS for mRCC between 2006 and 2015. SRS was indicated for alleviation of pain or neurological deficit or for local control of disease. Patients with emergent spinal instability, rapidly progressive neurological deficit, and less than 1 month of follow-up were excluded. Patients undergoing SRS were divided into 4 cohorts: those receiving concurrent first-line TKI therapy (A), systemic therapynaïve patients (B), and undergoing SRS with (C) or without (D) concurrent TKI therapy after failure of first-line TKI therapy. Patients were stratified to evaluate the effect of TKI-resistant and TKI-susceptible biology. Concurrent therapy was defined as TKIs administered on the day of SRS. Cohorts A-D were compared against a cohort (E) of patients with new or progressive spinal metastases managed with first-line TKI therapy alone; this cohort served as a negative control.

\section{Spine SRS}

SRS was conducted as described previously. ${ }^{3}$ Briefly, planning used CT simulation with $1.5-\mathrm{mm}$ slice thickness and high-definition MRI. Fusion of image sets was performed in BrainScan or iPlan software (BrainLab) or MIM (MIM Software Inc.). Target volumes were contoured according to RTOG $0631 .^{20,21}$ The spinal cord or cauda equina planning risk volume was contoured approximately $3 \mathrm{~mm}$ above and below the target volume. The spinal cord volume was constrained to $\mathrm{V}_{10}<10 \%$ and a maximum point dose of 14 Gy for single-fraction SRS; the cauda equina was constrained to $\mathrm{V}_{12}<10 \%$ and a maximum point dose of 16 Gy. Single-fraction prescription doses ranged from 10 to $18 \mathrm{~Gy}$, while hypofractionated regimens included 21 or $24 \mathrm{~Gy}$ in 3 fractions. Image guidance was achieved with cone-beam CT and/or the ExacTrac system (BrainLab). At 4-8 weeks following SRS, patients were evaluated in the clinic with spinal MRI, and then followed every 3 months thereafter. Patients experiencing a transient increase in pain within 10 days of SRS were considered to have pain flare.

\section{Local Control and Statistical Analysis}

The primary outcome was the 12-month cumulative incidence of local failure, which was assessed every 3 months with spinal MRI and calculated from the date of SRS (Cohorts A-D) or initiation of TKI therapy (Cohort E). Lesions demonstrating any in-field progression as evaluated by neuroradiologists were considered to represent local failure, regardless of post-SRS TKI cessation or initiation. This practice was standard throughout the study period. In Cohort E, local failure was only considered for progression of spine metastases present at the time of TKI initiation. Failures were categorized according to anatomical site (vertebral body, posterior elements, epidural space, neural foramen, and paraspinal tissues). Differences in demographic, disease, and dosimetric characteristics among cohorts were compared with Fisher's exact tests, Wilcoxon rank-sum tests, or ANOVA. Kaplan-Meier analysis was used to determine unadjusted overall survival, while cumulative incidence analysis was used to determine unadjusted local failure. Differences in overall survival and local failure were compared using log-rank and Gray's tests, respectively. Multivariate competing risks regression was used to control for the following covariates with death as a competing risk: performance status, symptomatology, systemic disease status, spinal disease burden, treatment-site location, number of treated vertebrae, epidural disease, neural foraminal involvement, paraspinal extension, prior irradiation, prior surgery, vertebral body fracture, and lesion maximum diameter. Covariates demonstrating association $(\mathrm{p} \leq 0.10)$ with local failure on univariate analysis were evaluated in a multivariate model. Covariate inclusion into the final multivariate model required $\mathrm{p} \leq 0.10$. Analyses were conducted using the R statistical software package (R Core Team 2015, R Foundation for Statistical Computing).

\section{Results}

\section{Patient and Treatment-Site Characteristics}

Among 854 SRS treatments (577 patients) in our institutional database, 151 treatments (100 patients) for mRCC were eligible for inclusion; 19 SRS treatments for mRCC were excluded because of follow-up duration of less than 1 month. Patient characteristics at the time of SRS are presented in Table 1. At the time of SRS, 70 patients (46\%) were receiving concurrent TKI treatment (Cohorts A and 
TABLE 1. Patient characteristics at spine SRS*

\begin{tabular}{|c|c|c|c|c|c|c|}
\hline Characteristic & Cohort A & Cohort B & Cohort C & Cohort D & Cohort E & $\mathrm{p}$ Value \\
\hline No. of treatment sites & 25 & 37 & 45 & 44 & 43 & \\
\hline Age (yrs) & & & & & & 0.14 \\
\hline Median & 57 & 61 & 61 & 58 & 59 & \\
\hline Range & $39-77$ & $39-87$ & $44-74$ & $47-80$ & $41-79$ & \\
\hline Male sex & $19(76)$ & $32(86)$ & $35(78)$ & $31(70)$ & $34(79)$ & 0.54 \\
\hline KPS & & & & & & 0.04 \\
\hline Median & 80 & 80 & 80 & 80 & 90 & \\
\hline Range & $50-90$ & $40-100$ & $60-90$ & $50-100$ & $60-90$ & \\
\hline NFS & & & & & & 0.30 \\
\hline Median & 1 & 1 & 1 & 1 & 1 & \\
\hline Range & $0-3$ & $0-3$ & $0-3$ & $0-3$ & $0-3$ & \\
\hline Prior smoker & $14(56)$ & $30(81)$ & $27(60)$ & $31(70)$ & $23(53)$ & 0.07 \\
\hline \multicolumn{7}{|l|}{ Comorbid disease } \\
\hline Hypertension & $14(56)$ & $29(78)$ & $31(69)$ & $34(77)$ & $31(72)$ & 0.34 \\
\hline Hyperlipidemia & $9(36)$ & $20(54)$ & $19(42)$ & $15(34)$ & $24(56)$ & 0.18 \\
\hline Diabetes & $4(16)$ & $10(27)$ & $6(13)$ & $9(20)$ & $10(23)$ & 0.58 \\
\hline Primary controlled & $19(76)$ & $24(65)$ & $43(96)$ & $37(84)$ & $35(81)$ & $<0.01$ \\
\hline \multicolumn{7}{|l|}{ Systemic disease characteristics } \\
\hline Solitary metastasis to spine & $2(8)$ & $9(24)$ & $3(7)$ & $4(9)$ & $4(9)$ & 0.16 \\
\hline Oligometastatic disease $(\leq 5)$ & $17(68)$ & $19(51)$ & $32(71)$ & $27(61)$ & $20(47)$ & 0.12 \\
\hline Extraspinal bone metastases & $22(88)$ & $16(43)$ & $32(71)$ & $27(61)$ & $23(53)$ & $<0.01$ \\
\hline Visceral metastases $†$ & $15(60)$ & $18(49)$ & $34(76)$ & $37(84)$ & $26(60)$ & $<0.01$ \\
\hline Brain metastases & $2(8)$ & $3(8)$ & $16(36)$ & $9(20)$ & $3(7)$ & $<0.01$ \\
\hline Spinal disease characteristics & & & & & & $<0.01$ \\
\hline Single-level disease & $4(16)$ & $11(30)$ & $6(13)$ & $8(18)$ & $21(49)$ & \\
\hline Multilevel disease (2-5 VBs) & $12(48)$ & $22(59)$ & $20(45)$ & $23(52)$ & $17(39)$ & \\
\hline Diffuse disease (>5 VBs) & $9(36)$ & $4(11)$ & $19(42)$ & $13(30)$ & $5(12)$ & \\
\hline No. of prior systemic agents & & & & & & $<0.01$ \\
\hline Median & 0 & 0 & 1 & 1 & 0 & \\
\hline Range & - & - & $1-8$ & $1-7$ & - & \\
\hline Radiographic follow-up (mos) & & & & & & $<0.01$ \\
\hline Median & 15 & 12 & 13 & 6 & 18 & \\
\hline Range & $1-68$ & $1-83$ & $1-48$ & $1-57$ & $1-84$ & \\
\hline Median overall survival (mos) & 18 & 21 & 16 & 8 & 24 & 0.02 \\
\hline Systemic agent at SRS & & & & & & $<0.01$ \\
\hline Sunitinib & $17(68)$ & $0(0)$ & $27(60)$ & 0 & $35(81)$ & \\
\hline Axitinib & $5(20)$ & $0(0)$ & $8(18)$ & 0 & $3(7)$ & \\
\hline Pazopanib & $2(8)$ & $0(0)$ & $6(13)$ & 0 & $3(7)$ & \\
\hline Sorafenib & $1(4)$ & $0(0)$ & $4(9)$ & 0 & $2(5)$ & \\
\hline Everolimus & $0(0)$ & $0(0)$ & $0(0)$ & $6(14)$ & $0(0)$ & \\
\hline Temsirolimusł & $0(0)$ & $0(0)$ & $0(0)$ & $2(5)$ & $0(0)$ & \\
\hline Bevacizumabł & $0(0)$ & $0(0)$ & $0(0)$ & $5(11)$ & $0(0)$ & \\
\hline No systemic therapy & $0(0)$ & $37(100)$ & $0(0)$ & $36(70)$ & $0(0)$ & \\
\hline
\end{tabular}

KPS = Karnofsky Performance Status; NFS = neurologic function score; VB = vertebral body.

* Data are presented as number (\%) unless otherwise indicated. Subtotals may exceed $100 \%$ (for example, if patients had multiple comorbidities). Cohort A comprised patients receiving concurrent first-line TKI therapy with SRS; Cohort B, systemic therapy-naïve patients undergoing SRS; Cohort C, patients undergoing SRS with concurrent TKI therapy after failure of first-line therapy; Cohort D, patients undergoing SRS without concurrent TKI therapy after failure of first-line systemic therapy; and Cohort E, patients with spinal metastases managed with first-line TKIs alone (negative control group).

$\dagger$ Organ other than bone or brain.

$\ddagger$ Within 30 days of SRS. 
TABLE 2. Treatment-site characteristics at SRS

\begin{tabular}{|c|c|c|c|c|c|c|}
\hline Characteristic & Cohort A & Cohort B & Cohort C & Cohort D & Cohort E & $p$ Value \\
\hline No. of treatment sites & 25 & 37 & 45 & 44 & 43 & \\
\hline Location & & & & & & 0.36 \\
\hline Cervical & $3(12)$ & $3(8)$ & $8(18)$ & $6(14)$ & $4(9)$ & \\
\hline Thoracic & $11(44)$ & $11(30)$ & $21(47)$ & $23(52)$ & $18(42)$ & \\
\hline Lumbosacral & $11(44)$ & $23(62)$ & $16(35)$ & $15(34)$ & $21(49)$ & \\
\hline Number of vertebral levels treated & & & & & & 0.03 \\
\hline 1 & $16(64)$ & $27(73)$ & $26(58)$ & $33(75)$ & $21(49)$ & \\
\hline 2 & $5(20)$ & $6(16)$ & $9(20)$ & $5(11)$ & $9(21)$ & \\
\hline$\geq 3$ & $4(16)$ & $4(11)$ & $10(22)$ & $6(14)$ & $13(30)$ & \\
\hline \multicolumn{7}{|l|}{ Treatment site characteristics } \\
\hline Epidural disease & $24(96)$ & $30(81)$ & $34(76)$ & $32(73)$ & $19(44)$ & $<0.01$ \\
\hline Neural foraminal involvement & $15(60)$ & $25(68)$ & $19(42)$ & $22(50)$ & $20(47)$ & 0.16 \\
\hline Posterior elements involvement & $12(48)$ & $14(38)$ & $22(49)$ & $24(55)$ & $15(35)$ & 0.34 \\
\hline Paraspinal extension & $10(40)$ & $15(41)$ & $16(36)$ & $22(50)$ & $13(30)$ & 0.42 \\
\hline Preexisting vertebral fracture & $8(32)$ & $13(35)$ & $12(27)$ & $10(23)$ & $15(35)$ & 0.67 \\
\hline Symptomatology & & & & & & 0.03 \\
\hline Pain alone & $16(64)$ & $22(60)$ & $34(76)$ & $34(77)$ & $28(65)$ & \\
\hline Pain w/ neurologic deficit & $7(28)$ & $3(8)$ & $2(4)$ & $3(7)$ & $4(9)$ & \\
\hline Asymptomatic & $2(8)$ & $12(32)$ & $9(20)$ & $7(16)$ & $11(26)$ & \\
\hline \multicolumn{7}{|l|}{ Prior local therapy } \\
\hline Any RT & $3(12)$ & $3(8)$ & $8(18)$ & $9(20)$ & $14(33)$ & 0.07 \\
\hline Any surgery & $7(28)$ & $8(22)$ & $5(11)$ & $6(14)$ & $13(30)$ & 0.12 \\
\hline Surgery $\mathrm{w} /$ instrumentation & $4(16)$ & $8(22)$ & $2(4)$ & $4(9)$ & $9(21)$ & 0.08 \\
\hline
\end{tabular}

$\mathrm{RT}=$ radiotherapy.

* Data are presented as number (\%). Subtotals may exceed $100 \%$ if a single lesion involved multiple anatomic sites. For cohort definitions, see Table 1 footnote or Methods.

C); among these, 25 (17\%) were receiving TKIs as firstline therapy (Cohort A). The relative distribution of patients among the 4 SRS cohorts was: A, 18 patients; B, 30 patients; C, 32 patients; and D, 28 patients. The incidence of patient crossover among cohorts was low (8\%), and there was no treatment-site crossover. The most common histology was clear-cell RCC (80\%). The median Karnofsky Performance Status was 80 for Cohorts A-D. Solitary metastasis to the spine was most common among therapy-naïve patients (Cohort $\mathrm{B}, \mathrm{p}=0.16)$. Patients who had failed first-line therapy (Cohorts $\mathrm{C}$ and $\mathrm{D})$ were more likely to have brain $(p<0.01)$ and visceral $(p<0.01)$ metastases as well as diffuse spinal disease $(p<0.01)$. The median overall survival after SRS (Cohorts A-D) or initiation of TKI treatment (Cohort E) was 8-24 months and was longest among patients in Cohorts $\mathrm{B}$ and $\mathrm{E}$. Pairwise comparisons between Cohorts A, B, C, and E with Cohort D were statistically significant $(p=0.02)$; all other pairwise comparisons were not statistically significant.

Treatment-site characteristics are reported in Table 2. A total of 232 vertebral levels were irradiated among 151 SRS treatments. The maximum number of treated vertebrae was 6 . Epidural disease was most common among patients receiving first-line TKIs concurrently with SRS (Cohort A, 96\%, $p=0.08$ ). A greater proportion of therapy-naïve patients (Cohort $\mathrm{B}$ ) were treated for local control alone $(32 \%, \mathrm{p}=0.03)$.
Dosimetric parameters are reported in Table 3. The median prescription dose was 16 Gy in a single fraction in each cohort. Two sites (1\%) were treated with a hypofractionated regimen ( 21 or 24 Gy in 3 fractions). Target volumes were slightly larger among therapy-naïve patients $(\mathrm{p}<0.01)$. Maximum tumor diameter was smallest among patients in Cohort E $(\mathrm{p}<0.01)$. Median treatment volume coverage was $93 \%-94 \%$ in each cohort $(\mathrm{p}=0.68)$.

\section{SRS Outcomes}

The cumulative incidence of local failure, sites of failure, and adverse events following SRS are presented in Table 4. At 12 months, the cumulative incidence of local failure was lowest (4\%) among patients treated with concurrent first-line TKI therapy (Cohort A, Fig. 1, p < 0.01). Twelve-month local failure was highest (57\%) among patients treated with TKIs alone (Cohort E). Local failure rates in Cohorts B (20\%), C (27\%), and D (19\%) were intermediate. Salvage surgery $(0 \%-8 \%)$ and irradiation $(4 \%-16 \%)$ were uncommon after SRS.

Adverse events following SRS were relatively modest. The combined incidence of pain flare was $17 \%$, which was similar across Cohorts A-D ( $\mathrm{p}=0.42)$, as was the incidence of post-SRS vertebral fracture $(21 \%, p=0.76)$. One patient in Cohort D experienced Grade 3 acute nausea/ vomiting after being treated simultaneously at 2 separate 
TABLE 3. Dosimetric parameters*

\begin{tabular}{|c|c|c|c|c|c|c|}
\hline Parameter & Cohort A & Cohort B & Cohort C & Cohort D & Cohort E & $p$ Value \\
\hline No. of treatment sites & 25 & 37 & 45 & 44 & 43 & \\
\hline No. of fractions (\%) & & & & & & 0.41 \\
\hline 1 & $25(100)$ & $37(100)$ & $45(100)$ & $42(95)$ & NA & \\
\hline 3 & $0(0)$ & $0(0)$ & $0(0)$ & $2(5)$ & NA & \\
\hline Prescription dose (Gy) & & & & & & 0.09 \\
\hline Median & 16 & 16 & 16 & 16 & NA & \\
\hline Range & $10-16$ & $14-18$ & $10-18$ & $10-24$ & NA & \\
\hline Target vol $\left(\mathrm{cm}^{3}\right)$ & & & & & & $<0.01$ \\
\hline Median & 66.1 & 87.6 & 59.8 & 51.0 & NA & \\
\hline Range & $25.6-529.0$ & $14.9-459.6$ & $3.3-1048.6$ & $9.0-422.2$ & NA & \\
\hline Max diameter $(\mathrm{cm})$ & & & & & & $<0.01$ \\
\hline Median & 7.3 & 7.5 & 5.9 & 6.0 & 5.1 & \\
\hline Range & $4.8-15.7$ & $3.1-14.2$ & $3.1-20.4$ & $1.1-11.4$ & $1.0-11.0$ & \\
\hline Max target dose (Gy) & & & & & & 0.09 \\
\hline Median & 18.2 & 18.2 & 18.1 & 17.7 & NA & \\
\hline Range & $11.2-20.9$ & $9.2-24.0$ & $11.5-23.8$ & $10.7-25.8$ & NA & \\
\hline Min target dose (Gy) & & & & & & 0.59 \\
\hline Median & 10.2 & 9.7 & 10.2 & 10.4 & NA & \\
\hline Range & $5.2-14.2$ & $4.8-13.5$ & $5.4-13.5$ & $6.3-22.9$ & NA & \\
\hline PTV coverage (\%) & & & & & & 0.68 \\
\hline Median & 94 & 93 & 93 & 94 & NA & \\
\hline Range & $90-99$ & $89-99$ & $79-100$ & $71-100$ & NA & \\
\hline Conformity index & & & & & & 0.06 \\
\hline Median & 1.4 & 1.2 & 1.4 & 1.3 & NA & \\
\hline Range & $1.0-1.9$ & $1.0-1.8$ & $1.1-2.4$ & $1.0-2.2$ & NA & \\
\hline Homogeneity index & & & & & & 0.09 \\
\hline Median & 1.2 & 1.2 & 1.2 & 1.1 & NA & \\
\hline Range & $1.1-1.3$ & $1.1-1.5$ & $1.1-1.3$ & $1.0-1.2$ & NA & \\
\hline
\end{tabular}

$\mathrm{NA}=$ not applicable PTV = planning target volume .

* For cohort definitions, see Table 1 footnote or Methods.

sites without a TKI. No other toxicity of Grade 3 or greater occurred following SRS.

Multivariate competing risks regression was conducted to adjust for differences among cohorts (Table 5). Several independent predictors of local failure were identified. Concurrent first-line TKI treatment (Cohort A) remained independently predictive of superior local control (HR $0.21, p=0.04$ ), while patients treated with TKIs alone (Cohort E) had the highest rate of local failure (HR 2.43, $\mathrm{p}=0.03$ ). Patients with multilevel disease experienced a higher rate of failure relative to single-level disease (HR $1.91, \mathrm{p}=0.02$ ), whereas patients with solitary metastases (HR 0.35, p = 0.04) had a lower rate of local failure.

\section{Discussion}

Many patients with $\mathrm{mRCC}$ receive TKIs shortly before, during, or after radiotherapy. Although there are reports of radiation dermatitis, bowel toxicities, and pneumonitis, TKIs are generally well tolerated when delivered with radiation. ${ }^{2,5,8,18,29}$ However, the safety and efficacy of concur- rent therapy remains particularly unknown despite a growing utilization of SRS in the mRCC patient population. Several investigators have postulated that TKIs offer an added benefit when delivered concurrently with radiation, extrapolating from preclinical models and the radiobiology of SRS. ${ }^{1,10,25,28}$ Because the interaction between targeted agents and radiation is poorly understood, clinicians may be hesitant to initiate TKIs until after completion of radiotherapy. Moreover, patients with solitary metastases and oligometastases are commonly managed with observation or SRS alone until development of greater disease burden. This population may benefit from up-front aggressive concurrent therapy.

In the present investigation, 151 spinal disease sites were treated with SRS at 232 vertebral levels. We compared local failure among cohorts of patients treated with or without concurrent TKI therapy, stratifying patients by failure of first-line therapy. To control for the independent effect of first-line TKI therapy, an additional cohort of patients who received TKIs without SRS served as a control group. We hypothesized that patients undergoing SRS with concurrent first-line TKI therapy achieve supe- 
TABLE 4. SRS outcomes*

\begin{tabular}{|c|c|c|c|c|c|}
\hline Outcome & Cohort A & Cohort B & Cohort C & Cohort D & Cohort E \\
\hline No. of treatment sites & 25 & 37 & 45 & 44 & 43 \\
\hline \multicolumn{6}{|c|}{ Local failure cumulative incidence } \\
\hline 6 month & $4 \%$ & $17 \%$ & $15 \%$ & $17 \%$ & $40 \%$ \\
\hline 12 month & $4 \%$ & $20 \%$ & $27 \%$ & $19 \%$ & $57 \%$ \\
\hline Sites of failure & $2(8)$ & $10(27)$ & $14(31)$ & $11(25)$ & $34(79)$ \\
\hline VB & $2(8)$ & $7(19)$ & $9(20)$ & $9(20)$ & $32(74)$ \\
\hline Epidural space & $1(4)$ & $6(16)$ & $12(27)$ & $8(16)$ & $16(37)$ \\
\hline Neural foramen & $0(0)$ & $4(11)$ & $5(11)$ & $3(7)$ & $16(37)$ \\
\hline Posterior elements & $0(0)$ & $2(5)$ & $3(7)$ & $2(5)$ & $14(33)$ \\
\hline Paraspinal & $0(0)$ & $3(8)$ & $3(7)$ & $3(7)$ & $13(30)$ \\
\hline Failure requiring surgery & $1(4)$ & $3(8)$ & $0(0)$ & $3(7)$ & $3(7)$ \\
\hline Failure requiring irradiation & $1(4)$ & $2(5)$ & $7(16)$ & $2(5)$ & $26(60)$ \\
\hline \multicolumn{6}{|l|}{ SRS-associated toxicities } \\
\hline Pain flare & $5(20)$ & $8(22)$ & $7(16)$ & $6(14)$ & NA \\
\hline Vertebral fracture $†$ & $6(27)$ & $10(27)$ & $8(18)$ & $6(14)$ & NA \\
\hline Grade $\geq 3$ toxicity & $0(0)$ & $0(0)$ & $0(0)$ & $2(5)$ & NA \\
\hline
\end{tabular}

* Data are presented as number (\%) unless otherwise indicated. Subtotals may exceed $100 \%$ if treatment sites had, e.g., multiple sites of failure. For cohort definitions, see Table 1 footnote or Methods.

$\dagger$ New or progressed fracture.

rior local control compared with other cohorts. Following multivariate analysis, a local control benefit was observed in support of this hypothesis (HR 0.21, p = 0.04, Fig. 1).

\section{Prior Literature}

Several investigations have reported noncomparative outcomes following stereotactic radiation with concurrent or adjuvant TKI administration. To our knowledge, no comparisons to SRS alone or TKI therapy alone have been reported, limiting the ability to interpret the results of these studies. In a retrospective study published in 2010, Staehler et al. ${ }^{24}$ analyzed outcomes for 106 patients with spinal (55 cases) or brain (51 cases) RCC metastases treated with SRS and concurrent sorafenib or sunitinib. The overall 15-month local control rate was 98\%; for spinal lesions, the 12- and 24-month local control rates were 94\% and $90 \%$. Competing risks were not considered when estimating local failure. No SRS-related toxicities of Grade 3 or greater were observed.

In 2014, Kao et al..$^{10}$ reported outcomes for 46 patients treated with concurrent sunitinib and fractionated stereotactic body radiotherapy (SBRT) for oligometastatic disease in a single-arm Phase I/II trial. No control cohort was used. All disease histologies and extracranial treatment sites were eligible, and only 7 patients (15\%) had mRCC.

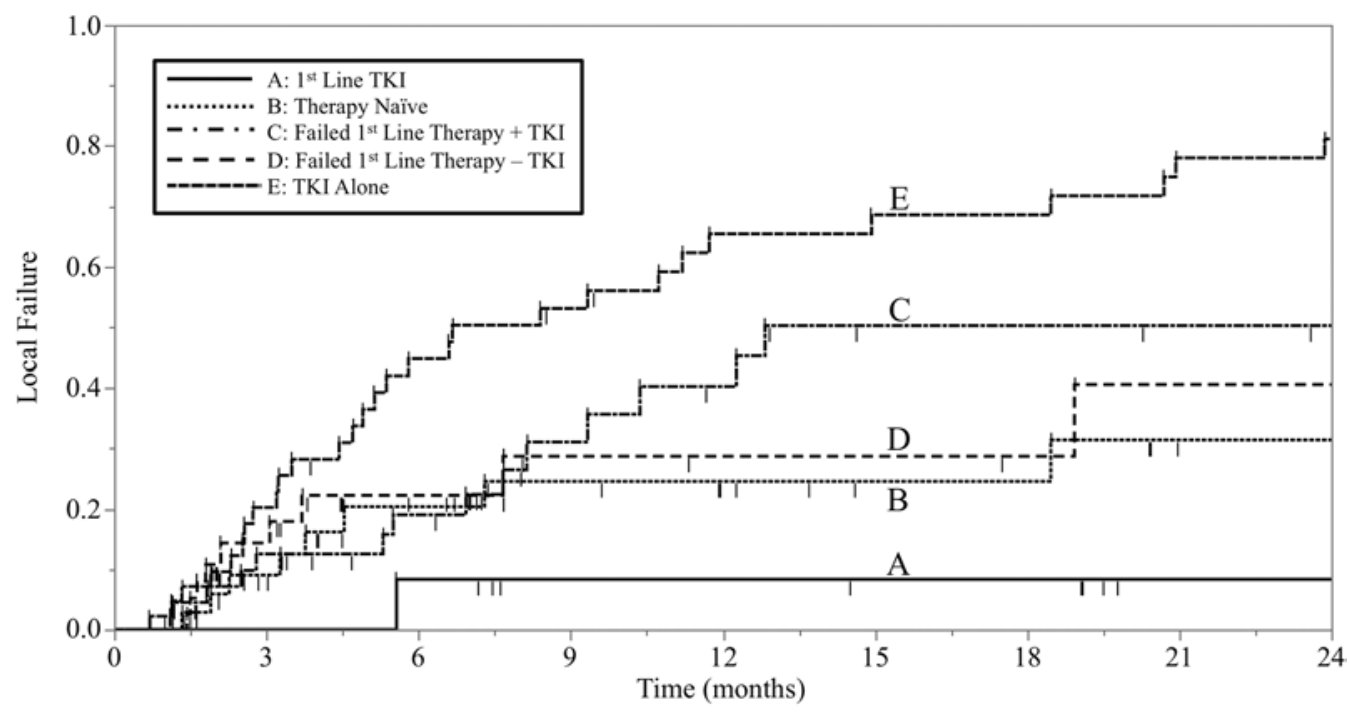

FIG. 1. Cumulative incidence of local failure in each cohort. Tick marks denote censoring. TKI = tyrosine kinase inhibitor. 
TABLE 5. Multivariate competing risks regression for local failure

\begin{tabular}{|c|c|c|c|c|}
\hline \multirow[b]{2}{*}{ Covariate } & \multirow{2}{*}{$\begin{array}{l}\text { Univariate } \\
\text { p Value }\end{array}$} & \multicolumn{3}{|c|}{ Multivariate* } \\
\hline & & p Value & $\mathrm{HR}$ & $95 \% \mathrm{Cl}$ \\
\hline KPS & 0.69 & - & - & - \\
\hline Symptomatology & & - & - & - \\
\hline Pain & 0.15 & - & - & - \\
\hline Pain + neurologic deficit & 0.39 & - & - & - \\
\hline Asymptomatic & [ref] & - & - & - \\
\hline \multicolumn{5}{|l|}{ Cohort } \\
\hline A (1st-line TKI + SRS) & 0.04 & 0.04 & 0.21 & $0.05-0.95$ \\
\hline B (therapy naïve + SRS) & [ref] & [ref] & 1.00 & - \\
\hline C (TKI + SRS after failure of 1st-line Tx) & 0.78 & 0.94 & 0.97 & $0.42-2.25$ \\
\hline D (SRS w/o TKI after failure of 1st-line Tx) & 0.82 & 0.58 & 0.78 & $0.31-1.92$ \\
\hline $\mathrm{E}$ (TKI alone) & $<0.01$ & 0.03 & 2.43 & $1.10-5.40$ \\
\hline \multicolumn{5}{|l|}{ Systemic disease characteristics } \\
\hline Solitary metastasis to spine & 0.07 & 0.04 & 0.35 & $0.13-0.95$ \\
\hline Oligometastatic disease ( $\leq 5$ metastases) & 0.01 & 0.61 & 0.87 & $0.51-1.49$ \\
\hline Extraspinal bone metastases & 0.11 & - & - & - \\
\hline Visceral metastases & 0.34 & - & - & - \\
\hline Multilevel disease ( $\geq 2 \mathrm{VBs}$ ) & 0.01 & 0.02 & 1.91 & $1.09-3.37$ \\
\hline \multicolumn{5}{|l|}{ Treatment site characteristics } \\
\hline \multicolumn{5}{|l|}{ Location } \\
\hline Cervical & [ref] & - & - & - \\
\hline Thoracic & 0.27 & - & - & - \\
\hline Lumbosacral & 0.19 & - & - & - \\
\hline Prior RT & 0.83 & - & - & - \\
\hline Prior surgery & 0.56 & - & - & - \\
\hline Epidural disease & 0.09 & 0.45 & 1.19 & $0.75-1.89$ \\
\hline Neural foraminal involvement & 0.30 & - & - & - \\
\hline Paraspinal extension & 0.95 & - & - & - \\
\hline Baseline VB fracture & 0.31 & - & - & - \\
\hline No. of treated levels & $<0.01$ & 0.10 & 1.15 & $0.97-1.36$ \\
\hline Max diameter $(\mathrm{cm})$ & 0.23 & - & - & - \\
\hline
\end{tabular}

The actuarial 2-year local control rate was 75\%. No SBRTassociated toxicities of Grade 3 or greater occurred; however, 1 case of fatal hemoptysis occurred after sunitinib initiation in a patient previously treated with head and neck radiation. Following SBRT, 33\% of patients experienced 1 or more toxicities of Grade 3 or greater attributed to sunitinib.

In the present investigation, the cumulative incidence of 12-month local failure (4\%) in Cohort A was similar to that observed by Staehler et al. (6\%). In comparison, local control for mRCC spine metastases treated with singlefraction SRS has been reported to be $71 \%$ at 12 months. ${ }^{3}$ The present investigation controlled for differences in demographics, prior local therapy, and systemic and spinal disease burden in an effort to identify the independent effect of concurrent first-line TKI treatment upon SRS. In this cohort, no patient with solitary metastases or oligometastases experienced local failure. Notably, this response was not observed for patients who underwent SRS after failure of first-line therapy despite receiving concurrent TKI therapy (Cohort C). This may relate to the biology of TKI-resistant metastases and suggests that TKIs may not improve the efficacy of SRS in this setting.

Patients in Cohort A achieved superior local control (HR 0.21, p = 0.04) compared with therapy-naïve patients (Cohort B), despite a lower proportion of solitary metastases $(8 \%$ vs $24 \%)$. For patients with solitary metastases, treatment may be curative, with multivariate analysis demonstrating a favorable response to SRS (HR 0.35, p = 0.04). However, we did consider that the local control benefit observed in Cohort A was attributable to first-line TKI treatment alone. In an effort to study this effect, a control cohort of patients with new or progressive spinal metastases managed with first-line TKIs was included (Cohort E). This cohort, albeit with a greater number of treated metastases, demonstrated the highest local failure rate (HR 
2.43, $\mathrm{p}=0.03$ ), suggesting that SRS does indeed offer a benefit beyond first-line TKI treatment alone. This is supported by the fact that the observed 12-month local control rate in Cohort E (43\%) is similar to the progression-free survival rate $(25 \%)$ observed in a large randomized trial comparing first-line sunitinib to interferon-alfa. ${ }^{17}$ Although control of spinal disease certainly differs from control of systemic disease, these data suggest that the control cohort approximates the expected benefit of systemic therapy alone. Consequently, the addition of concurrent first-line TKI therapy to SRS in the oligometastatic setting could result in a progression-free survival benefit and would warrant further study. Indeed, the 24-month local control rate among patients with solitary metastases or oligometastases in Cohort A was 100\%, compared with $75 \%$ in Cohort B and $20 \%$ in Cohort $\mathrm{E}(\mathrm{p}<0.01)$.

Crossover among cohorts following SRS would certainly occur in this population. In particular, therapy-naïve patients would eventually undergo systemic therapy and receive TKIs. TKIs may offer a benefit as adjuvant treatment following radiation, and therefore this cohort biases our results toward the null hypothesis. Furthermore, patients managed with TKIs alone (Cohort E) may eventually undergo spinal radiotherapy, further biasing these results toward the null. Therefore, the presence of crossover strengthens our findings and suggests that concurrent TKI treatment does indeed offer an added benefit. These results are perhaps explained by the antiangiogenic mechanism of TKIs in light of the current understanding of hypofractionated radiotherapy and its effect on vasculature. ${ }^{4}$ Moreover, the added benefit of TKIs may eliminate the need for SRS dose-escalation beyond $20 \mathrm{~Gy}$, which is associated with a significantly increased risk for compression fracture and toxicity of Grade 3 or greater., ${ }^{7,22}$

Although significant differences in favorable and unfavorable characteristics were present among our cohorts, patients in Cohort A did not have characteristics that would traditionally favor superior local control. A relatively high prevalence of diffuse spinal disease (36\%) was present, and several treatment sites $(16 \%)$ involved treatment of more than 2 vertebral levels. Moreover, Cohort A had the highest prevalence of epidural disease $(96 \%, \mathrm{p}=0.08)$, a known risk factor for local failure.

Importantly, the use of concurrent TKI therapy with SRS was not associated with an increased risk for toxicity. One patient not receiving a TKI suffered from acute Grade 3 nausea and vomiting. No other patient experienced toxicity of Grade 3 or greater attributable to SRS. In addition, the rates of pain flare and vertebral fracture were not significantly elevated among patients receiving concurrent SRS and TKI therapy and were comparable to rates previously reported in the literature. ${ }^{9,22}$

\section{Limitations}

Several limitations must be considered when interpreting these results. Although patients receiving first-line TKI therapy experienced a benefit with regard to local control (Cohort A), this was not true for patients who were treated after failure of first-line therapy despite receiving TKIs concurrently with SRS (Cohort C). This observation suggests that metastatic disease refractory to first-line therapy responds similarly to SRS, even in the presence of a TKI. It is possible that these observations result from either downregulation or differential expression of VEGF receptors among TKI-sensitive and TKI-resistant patients.

As a retrospective investigation, these results are subject to selection and measurement biases. In an effort to address this, 4 control cohorts were used to test our hypothesis, and consecutive patients were included. Moreover, SRS planning and delivery across treatment sites were extremely uniform. A further limitation is the difference in survival among cohorts: this limits the opportunity to draw longterm conclusions regarding efficacy of therapy.

Utilization of controls distinguishes the present study from prior investigations, which have generally been single-arm retrospective or prospective investigations evaluating toxicity and feasibility rather than efficacy. Although certain characteristics differed among cohorts, an effort to control for confounding differences was made with multivariate analysis. Both unadjusted and adjusted analyses supported the research hypothesis.

\section{Conclusions}

The prognosis for patients with $\mathrm{mRCC}$ has significantly improved with the development of small-molecule TKIs. As the prevalence of this disease increases, the demand for radiation therapy similarly increases in an effort to alleviate symptoms and delay second-line therapy. Although intended to control systemic disease, TKIs may offer an added local control benefit when delivered concurrently with radiation. In the present investigation, we observed a significant and independent local control benefit with the addition of first-line TKIs to spine SRS. These results have implications in the oligometastatic setting and offer clinical evidence to support a growing body of radiobiological research for the utilization of small-molecule TKIs concurrently with high-dose, hypofractionated radiotherapy.

\section{References}

1. Ahluwalia MS, Chao ST, Parsons MW, Suh JH, Wang D, Mikkelsen T, et al: Phase II trial of sunitinib as adjuvant therapy after stereotactic radiosurgery in patients with 1-3 newly diagnosed brain metastases. J Neurooncol 124:485-491, 2015

2. Azad A, Maddison C, Stewart J: Radiation recall dermatitis induced by pazopanib. Onkologie 36:674-676, 2013

3. Balagamwala EH, Angelov L, Koyfman SA, Suh JH, Reddy CA, Djemil T, et al: Single-fraction stereotactic body radiotherapy for spinal metastases from renal cell carcinoma. J Neurosurg Spine 17:556-564, 2012

4. Balagamwala EH, Chao ST, Suh JH: Principles of radiobiology of stereotactic radiosurgery and clinical applications in the central nervous system. Technol Cancer Res Treat 11:3-13, 2012

5. Basille D, Andrejak M, Bentayeb H, Kanaan M, Fournier C, Lecuyer E, et al: Bronchial fistula associated with sunitinib in a patient previously treated with radiation therapy. Ann Pharmacother 44:383-386, 2010

6. Chang EL, Shiu AS, Mendel E, Mathews LA, Mahajan A, Allen PK, et al: Phase I/II study of stereotactic body radiotherapy for spinal metastasis and its pattern of failure. J Neurosurg Spine 7:151-160, 2007

7. Cox BW, Jackson A, Hunt M, Bilsky M, Yamada Y: Esophageal toxicity from high-dose, single-fraction paraspinal 
stereotactic radiosurgery. Int J Radiat Oncol Biol Phys 83:e661-e667, 2012

8. Inoue T, Kinoshita H, Komai Y, Kawabata T, Kawa G, Uemura Y, et al: Two cases of gastrointestinal perforation after radiotherapy in patients receiving tyrosine kinase inhibitor for advanced renal cell carcinoma. World J Surg Oncol 10:167, 2012

9. Jung DL, Balagamwala EH, Angelov L, Suh JH, Reddy CA, Djemil T, et al: Incidence and risk factors for pain flare following spine radiosurgery. Int J Radiat Oncol Biol Phys 87 Suppl:S568-S569, 2013 (Abstract)

10. Kao J, Chen CT, Tong CCL, Packer SH, Schwartz M, Chen $\mathrm{SH}$, et al: Concurrent sunitinib and stereotactic body radiotherapy for patients with oligometastases: final report of a prospective clinical trial. Target Oncol 9:145-153, 2014

11. Koyfman SA, Djemil T, Burdick MJ, Woody N, Balagamwala $\mathrm{EH}$, Reddy CA, et al: Marginal recurrence requiring salvage radiotherapy after stereotactic body radiotherapy for spinal metastases. Int J Radiat Oncol Biol Phys 83:297-302, 2012

12. Lam JS, Leppert JT, Belldegrun AS, Figlin RA: Novel approaches in the therapy of metastatic renal cell carcinoma. World J Urol 23:202-212, 2005

13. Mauceri HJ, Hanna NN, Beckett MA, Gorski DH, Staba MJ, Stellato KA, et al: Combined effects of angiostatin and ionizing radiation in antitumour therapy. Nature 394:287-291, 1998

14. Motzer RJ, Bander NH, Nanus DM: Renal-cell carcinoma. N Engl J Med 335:865-875, 1996

15. Motzer RJ, Hutson TE, Cella D, Reeves J, Hawkins R, Guo J, et al: Pazopanib versus sunitinib in metastatic renal-cell carcinoma. N Engl J Med 369:722-731, 2013

16. Motzer RJ, Hutson TE, Tomczak P, Michaelson MD, Bukowski RM, Oudard S, et al: Overall survival and updated results for sunitinib compared with interferon alfa in patients with metastatic renal cell carcinoma. J Clin Oncol 27:35843590, 2009

17. Motzer RJ, Hutson TE, Tomczak P, Michaelson MD, Bukowski RM, Rixe O, et al: Sunitinib versus interferon alfa in metastatic renal-cell carcinoma. N Engl J Med 356:115-124, 2007

18. Neri A, Lambert Y, Marrelli D, Di Mare G, Mastrogiacomo $\mathrm{D}$, Corso G, et al: Gastro-pleuro-pericardial fistula following combined radiation and chemotherapy for lung metastases from renal cell carcinoma: report of a case. Surg Today 43:1457-1460, 2013

19. Nguyen QN, Shiu AS, Rhines LD, Wang H, Allen PK, Wang $X S$, et al: Management of spinal metastases from renal cell carcinoma using stereotactic body radiotherapy. Int J Radiat Oncol Biol Phys 76:1185-1192, 2010

20. Ryu S, Pugh SL, Gerszten PC, Yin FF, Timmerman RD, Hitchcock YJ, et al: RTOG 0631 Phase $2 / 3$ study of image guided stereotactic radiosurgery for localized (1-3) spine metastases: Phase 2 results. Pract Radiat Oncol 4:76-81, 2014

21. Ryu S, Pugh SL, Gerszten PC, Yin FF, Timmerman RD, Hitchcock YJ, et al: RTOG 0631 Phase II/III study of imageguided stereotactic radiosurgery for localized (1-3) spine metastases: Phase II results. Int J Radiat Oncol Biol Phys 81:S131-S132, 2011

22. Sahgal A, Atenafu EG, Chao S, Al-Omair A, Boehling N, Balagamwala EH, et al: Vertebral compression fracture after spine stereotactic body radiotherapy: a multi-institutional analysis with a focus on radiation dose and the spinal instability neoplastic score. J Clin Oncol 31:3426-3431, 2013

23. Schueneman AJ, Himmelfarb E, Geng L, Tan J, Donnelly E, Mendel D, et al: SU11248 maintenance therapy prevents tu- mor regrowth after fractionated irradiation of murine tumor models. Cancer Res 63:4009-4016, 2003

24. Staehler M, Haseke N, Nuhn P, Tüllmann C, Karl A, Siebels $\mathrm{M}$, et al: Simultaneous anti-angiogenic therapy and singlefraction radiosurgery in clinically relevant metastases from renal cell carcinoma. BJU Int 108:673-678, 2011

25. Straka C, Kim DWN, Timmerman RD, Pedrosa I, Jacobs C, Brugarolas J: Ablation of a site of progression with stereotactic body radiation therapy extends sunitinib treatment from 14 to 22 months. J Clin Oncol 31:e401-e403, 2013

26. Thibault I, Al-Omair A, Masucci GL, Masson-Côté L, Lochray F, Korol R, et al: Spine stereotactic body radiotherapy for renal cell cancer spinal metastases: analysis of outcomes and risk of vertebral compression fracture. J Neurosurg Spine 21:711-718, 2014

27. Vickers MM, Al-Harbi H, Choueiri TK, Kollmannsberger C, North S, MacKenzie M, et al: Prognostic factors of survival for patients with metastatic renal cell carcinoma with brain metastases treated with targeted therapy: results from the international metastatic renal cell carcinoma database consortium. Clin Genitourin Cancer 11:311-315, 2013

28. Wuthrick EJ, Curran WJ Jr, Camphausen K, Lin A, Glass J, Evans J, et al: A pilot study of hypofractionated stereotactic radiation therapy and sunitinib in previously irradiated patients with recurrent high-grade glioma. Int J Radiat Oncol Biol Phys 90:369-375, 2014

29. Yuasa T, Kitsukawa S, Sukegawa G, Yamamoto S, Kudo K, Miyazawa K, et al: Early onset recall pneumonitis during targeted therapy with sunitinib. BMC Cancer 13:3, 2013

30. Zhu H, Wang Z, Xu Q, Zhang Y, Zhai Y, Bai J, et al: Inhibition of STAT1 sensitizes renal cell carcinoma cells to radiotherapy and chemotherapy. Cancer Biol Ther 13:401-407, 2012

\section{Disclosures}

Dr. Suh reports receiving support for travel and lodging and/ or research from Elekta and Varian Medical Systems. Dr. Rini reports a consultant relationship with Pfizer. Dr. Garcia reports a consultant relationship with Astellas Pharma US, Inc.; Bayer Healthcare Pharmaceuticals, Inc.; Pfizer, Inc.; and Sanofi S.A. Dr. Ahluwalia reports a consultant relationship with Incyte Corp. and PrIME Oncology, Inc. Dr. Chao reports honorarium receipt from Varian.

\section{Supplemental Information}

Previous Presentations

Portions of this work were presented during the plenary session of the American Association of Neurological Surgeons annual meeting, Chicago, Illinois, May 4, 2016.

\section{Author Contributions}

Conception and design: Miller. Acquisition of data: Miller, Balagamwala. Analysis and interpretation of data: Miller. Drafting the article: Miller, Balagamwala. Critically revising the article: all authors. Reviewed submitted version of manuscript: all authors. Statistical analysis: Miller. Study supervision: Chao.

\section{Correspondence}

Samuel T. Chao, Department of Radiation Oncology, Desk T28, Taussig Cancer Institute, Cleveland Clinic, Cleveland, OH 44195, Email: chaos@ccf.org. 